\title{
Desarrollo e implementación de un blog colaborativo como instrumento de coevaluación en el Grado de Veterinaria
}

\section{Development and implementation of a collaborative blog as a co-assessment instrument in Veterinary Medicine Degree}

\author{
Nahúm Ayala Soldado ${ }^{1}$, Rafael Mora Medina ${ }^{2}$, Antonio Jesús Lora Benítez ${ }^{3}$, \\ Ana $M^{a}$ Molina López ${ }^{4}, \& M^{a}$ del Rosario Moyano Salvago ${ }^{5}$
}

Fecha de recepción: 19/11/2019; Fecha de revisión: 13/01/2020; Fecha de aceptación: 18/02/2020

Cómo citar este artículo:

Ayala, N., Mora, R., Lora, A.J., Molina, A. Ma \& Moyano, Mํㅡ R. (2020). Desarrollo e implementación de un blog colaborativo como instrumento de coevaluación en el Grado de Veterinaria. Revista de Innovación y Buenas Prácticas Docentes, 9(1), 61-70.

Autor de Correspondencia: nahum.ayala@uco.es

\section{Resumen:}

La evaluación educativa pretende comprometer al alumnado en su proceso de enseñanza-aprendizaje mediante el uso de estrategias e instrumentos de coevaluación y autoevaluación. En este sentido, las tecnologías de la información y de la comunicación (TIC) promueven un clima de enseñanza más dinámico a través del aprendizaje activo, siendo los blogs colaborativos una herramienta muy útil de evaluación del alumnado. El objetivo de este proyecto ha sido evaluar la implementación de un blog como instrumento educativo para promover la autorregulación por medio de actividades de coevaluación y/o autoevaluación en el ámbito universitario. Se diseñó un blog de divulgación científica como recurso docente para la asignatura optativa de "el Animal de Laboratorio", perteneciente al Grado de Veterinaria. Se evaluó mediante cuestionarios tanto la situación inicial del alumnado, como los logros finales respecto al objetivo planteado, siguiendo una metodología descriptiva. Según los resultados obtenidos, es innegable decir que el uso del blog ha sido una práctica innovadora, siendo en muchos casos la primera experiencia con TIC para el alumnado. Respecto a su implementación, los resultados han sido satisfactorios para la mejora motivacional, el aprendizaje y la dinamización metodológica. Todo ello, pone de manifiesto las múltiples ventajas que ofrecen los blogs como herramienta de coevaluación.

Palabras clave: Evaluación formativa, Autoevaluación, Tecnologías de la Información y de la Comunicación, Motivación

\footnotetext{
Abstract:

The educational evaluation aims to engage students in their teaching-learning process through the use of strategies and instruments of co-assessment and self-assessment In this sense, information and communication technologies (ICT) promote a more dynamic teaching environment through active learning, and collaborative blogs appear as a very useful tool for student assessment. The aim of this project has been to evaluate the implementation of a blog as an educational instrument to promote self-regulation through co-evaluation and / or self-evaluation activities at the university. To this end, a scientific dissemination blog was designed as a teaching resource for the optional subject "the Laboratory Animal", belonging to the Veterinary Science Degree syllabus. The starting point of the learning process of the students was evaluated by questionnaires, as well as the final achievements related to the proposed objective, following a descriptive methodology. According to the obtained results, it is undeniable that the development of the blog has been an innovative practice, being in many cases the first experience with ICT for students. Regarding its implementation, results suggest that students' motivation has improved,

1 Universidad de Córdoba (España), nahum.ayala@uco.es; CÓDIGO ORCID: 0000-0003-4534-7545

${ }^{2}$ Universidad de Córdoba (España), v02momer@uco.es; CÓDIGO ORCID: 0000-0001-6525-3381

${ }^{3}$ Universidad de Córdoba (España), v12lobea@uco.es; CÓDIGO ORCID: 0000-0001-7098-8606

${ }^{4}$ Universidad de Córdoba (España), ft2moloa@uco.es; CÓDIGO ORCID: 0000-0002-2645-7303

${ }^{5}$ Universidad de Córdoba (España), r.moyano@uco.es; CÓDIGO ORCID: 0000-00034705-0307
} 
and the learning procedure and methodologies applied have been dynamized. All this shows the many advantages that blogs offer as a co-assessment tool.

Key Words: Formative evaluation, Self-evaluation, Information and Communication Technologies, Motivation 


\section{INTRODUCCIÓN}

Actualmente, en el marco de la formación por competencias, la evaluación se define como un proceso continuo y sistemático de recogida y análisis de información orientado a la toma de decisiones para la mejora de los procesos de enseñanzaaprendizaje. La denominada "evaluación educativa" es un elemento clave para comprometer al alumnado en la participación del control y la responsabilidad de su proceso de enseñanza-aprendizaje mediante el uso de estrategias e instrumentos de evaluación mutua, coevaluación y autoevaluación. De esta manera, el estudiante debe reflexionar acerca del desarrollo de su propio proceso, de sus éxitos y de sus fracasos, lo que solamente se consigue cuando cada alumno se corresponsabiliza de su propio progreso (Brown \& Glasner, 2003).

Dicha evaluación educativa proporciona múltiples ventajas al alumno, aportándole estrategias para su desarrollo personal y profesional, de manera que ayuda a desarrollar su capacidad crítica, favorece su autonomía en el proceso educativo, lo motiva y responsabiliza en el aprendizaje, promueve su capacidad crítica y fomenta el desarrollo de aspectos sociales, tales como la convivencia, solidaridad, sociabilidad, respeto mutuo, etc. Por otro lado, a los docentes les ayuda a tomar conciencia respecto a los avances y problemas individuales y grupales; asimismo, les permite valorar la actuación de los alumnos en el grupo y facilitar la reorientación de sus procesos de aprendizaje. En base a esto, la actividad del profesorado debe estar orientada a desarrollar en el alumnado un aprendizaje que les proporcione autonomía e independencia parcial. Para ello, los docentes deben fomentar estrategias que propicien la adquisición de las competencias, de forma que sean capaces de "aprender a aprender" (González, 2003; Stowell, 2004).

El método de evaluación del aprendizaje del alumnado es objeto de debate continuo, muestra de ello son el gran número de investigaciones que se han realizado al respecto. Muchas de ellas han puesto de manifiesto que la forma de evaluación "tradicional" está desequilibrada, limitándose ésta, normalmente, a la emisión de una calificación, sin profundizar en los procesos que han desembocado en dichos resultados, y obviando, por lo tanto, la perspectiva del alumnado (Darby, 2007).

En el ámbito universitario esta problemática es de gran interés debido a la emergencia de una nueva forma renovada de evaluación centrada en las necesidades educativas de los estudiantes. Actualmente, los títulos universitarios se basan en el "European Credit Transfer System" (ECTS) que propone medir, no solo los resultados académicos, sino el tiempo dedicado al estudio (González, 2006). En este sentido, son numerosos los autores que manifiestan la necesidad de promover en las universidades un clima de enseñanza más dinámico, a través, por ejemplo, del aprendizaje activo y cooperativo basado en problemas, fomentando el uso de las tecnologías de la información y de la comunicación (TIC). De esta forma, se han desarrollado un conjunto de procedimientos bajo la denominación de "evaluación alternativa", donde el aprendizaje se realiza a través de tareas reales (González, 2003; Jackson \& Ward, 2004).

El uso de las TIC posibilita disponer de un volumen casi ilimitado de información a tiempo real, que se modifica y actualiza de manera constante. Dentro de las posibilidades que nos ofrecen, los blogs colaborativos pueden ser una herramienta 
sencilla, fácil de implementar y muy útil a la hora de evaluar el aprendizaje del alumnado. En este sentido, su utilización en las aulas como herramienta pedagógica, permite al alumnado centrarse, no solo en los contenidos, sino también en el proceso de comunicación, fomentando la participación a través del diálogo (Gamliel et al., 2005; Huffaker, 2005). Por ejemplo, en experiencias previas realizadas en asignaturas del Grado de Enfermería de la Universidad de Castilla-La Mancha, se evaluó el uso de blogs de manera positiva desde una perspectiva teórica y desde el punto de vista de los evaluadores, reproduciéndose la mayoría de las ventajas expuestas anteriormente (Conde-Caballero et al., 2019).

Esta herramienta permite la orientación y evaluación objetiva en la práctica educativa. De manera que el estudiante puede llevar a cabo una autoevaluación y coevaluación, mientras que al docente le permite aplicar una heteroevaluación mediante pautas que previamente han sido acordadas, consensuadas y expuestas sobre los criterios que se van a evaluar. Así pues, el empleo de blogs grupales no solo favorece una evaluación más sistematizada por parte del docente, sino que son una herramienta de extraordinario valor para el desarrollo de competencias de monitorización, autoevaluación y evaluación entre pares, contribuyendo a un mayor entendimiento y control del propio proceso de enseñanza-aprendizaje (Delgado, 2018).

El objetivo de este proyecto ha sido evaluar la implementación de un blog colaborativo para la asignatura optativa del Grado en Veterinaria de "el Animal de laboratorio", como instrumento educativo que promueva la autorregulación por medio de actividades de coevaluación y/o autoevaluación.

\section{DESARROLLO DE LA EXPERIENCIA DE INNOVACIÓN}

\subsection{Población de estudio y contexto educativo}

El presente proyecto se diseñó para el alumnado de la asignatura optativa de "el Animal de Laboratorio", perteneciente al 5ำ curso del Grado de Veterinaria, durante el curso académico 2018/19, siendo 34 el total de alumnos matriculados en dicho curso académico. Esta asignatura pretende dar una visión más clara del uso de animales como modelos de estudio en biomedicina, ofreciendo una perspectiva objetiva del aporte que suponen, y de las ventajas e inconvenientes de su utilización. La asignatura consta de 3 créditos ECTS ( 30 horas de trabajo presencial y 45 horas de trabajo no presencial). En lo que a metodología se refiere, se evalúan habilidades como la resolución de problemas, el trabajo en equipo, la capacidad de aplicar los conocimientos a la práctica y el conocimiento y aplicación práctica de los principios y metodologías de la veterinaria, así como la adquisición de las destrezas y competencias descritas en los objetivos generales del título, tratando temas como la protección animal, la bioética o la comunicación y divulgación de la ciencia.

\subsection{Acción metodológica}

Para el proyecto se diseñó y desarrolló el blog de la asignatura, "La cuarta $R$ ", alojado en el sistema de gestión de contenidos WordPress. Las instrucciones para inscribirse en el mismo y acceder como colaboradores fueron proporcionadas al alumnado a través de la plataforma Moodle de la asignatura, mostrando tanto su contenido como su funcionamiento. La primera vez que se accedía a la web, los alumnos debían registrarse con un correo electrónico y una contraseña personal para acceder posteriormente y poder ser identificados.

Desde el punto de vista metodológico, este proyecto puede dividirse en tres momentos de desarrollo: 
- Fase inicial. Consistió en una evaluación inicial de la experiencia previa que tenía cada estudiante en el ámbito del uso de los blogs u otras herramientas tecnológicas, con el fin de enseñar su funcionamiento, solventar posibles dudas, y motivar a los alumnos para la redacción de sus propias entradas. En este sentido, en las primeras sesiones se llevó a cabo un cuestionario inicial. Igualmente, el profesorado expuso los objetivos de aprendizaje y contenidos más relevantes, así como los indicadores básicos de calidad de la tarea, siendo éstos los aspectos fundamentales:

a. Escribir una entrada de carácter divulgativo en el blog grupal de la asignatura.

b. Seleccionar una temática de interés y actualidad relacionada con el contenido de la asignatura: cuestiones bioéticas, el bienestar animal, las buenas prácticas científicas y la aplicación de las 'tres erres'.

c. Participación activa en el blog: leer y comentar de manera constructiva y respetuosa las entradas del resto de compañeros y compañeras.

d. Tutorización y seguimiento por parte del profesorado.

e. Fechas y plazos de interés.

- Fase de desarrollo. La segunda fase se correspondió con el desarrollo de la tarea propuesta, es decir, la redacción de una entrada divulgativa en el blog de la asignatura y posterior lectura e interacción en las entradas del resto de compañeros y compañeras, fomentando de esta forma el debate. Para ello, se expusieron los aspectos fundamentales a tener en cuenta a la hora de desarrollar un texto científico de carácter divulgativo: temática y formato, origen y gestión de la información o fomento y promoción del interés y la participación, entre otros. Esta fase fue dirigida en todo momento por el profesorado, potenciando la utilización de distintas técnicas e instrumentos de trabajo, bibliografía complementaria, etc., teniendo en cuenta las posibles dificultades que pudieran darse, así como proponiendo fórmulas para evitarlas.

- Fase de coevaluación. En esta última etapa del proyecto, y de manera conjunta, docentes y estudiantes analizaron los objetivos alcanzados por el grupo. Lo cual permitió al profesorado orientar a los estudiantes sobre sus dificultades y áreas susceptibles de mejora. En este sentido, se elaboró un cuestionario de autoevaluación del alumnado, que permitió determinar el grado de satisfacción alcanzado, así como implementar posibles acciones de mejora de la tarea realizada, llevando a cabo una evaluación de la evaluación (metaevaluación) contemplada como forma de avanzar en el perfeccionamiento profesional y en la mejora del proceso de enseñanzaaprendizaje.

La evaluación de los resultados tanto del cuestionario inicial, como del cuestionario de autoevaluación final son los que se exponen en este trabajo.

\subsection{Diseño de las encuestas}

Los cuestionarios empleados para el proyecto fueron diseñados específicamente para el desarrollo del mismo utilizando los formularios de Google. Los participantes accedían a ellos a través del enlace facilitado y sus respuestas se enviaban de forma anónima. 
Dichas encuestas se componían de varias preguntas, algunas de respuesta múltiple y otras de respuesta abierta, donde los alumnos podían exponer su propia opinión. En lo que se refiere a su estructura, ambas encuestas contenían una parte inicial con preguntas generales tales como la edad, el sexo, etc. El resto de cuestiones estaban relacionadas con el uso del blog de la asignatura como recurso educativo desde el punto de vista metodológico y específicamente, como instrumento de evaluación. Haciendo referencia dichas cuestiones a asuntos tales como la dedicación requerida, el sistema de aprendizaje o la metodología empleada, estableciendo además, comparativas entre el blog y otros métodos de trabajo tradicionales.

\subsection{Análisis estadístico de los resultados}

Para el análisis de los datos se ha usado el programa estadístico IBM SPSS en su versión 25. Dado el carácter de este estudio y la muestra que se ha utilizado, los datos que se presentan son de tipo cualitativo y cuantitativo, mostrando los resultados de manera descriptiva. Se realizó la prueba no paramétrica de Suma de rangos Wilcoxon para comparar las variables estudiadas antes y después de la implementación del blog, aceptando como significativo un valor de $p \leq 0,05$.

\section{RESULTADOS}

\subsection{Población de estudio}

La muestra de estudio $(n=34)$, se corresponde con el número de estudiantes matriculados en la asignatura de "el Animal de Laboratorio" en el curso 2018/19. La distribución de la muestra en relación con el sexo fue homogénea, siendo un 55,9\% alumnas frente a un $44,1 \%$ de alumnos. Por otro lado, la edad media de los participantes fue de 24 años.

\subsection{El uso del blog colaborativo como instrumento de evaluación}

A continuación, se exponen los principales resultados obtenidos en relación con el uso del blog colaborativo como instrumento de evaluación. En este sentido, cabe destacar los resultados de la pregunta relacionada con el uso de nuevos recursos educativos (blogs, redes sociales, foros, etc.) a lo largo de la formación universitaria, los cuales evidenciaron lo novedoso de este tipo de recursos dentro de la docencia, ya que un porcentaje acumulado del $61,8 \%$ de los alumnos "nunca" o "casi nunca" los había utilizado en las asignaturas del Grado (tabla 1).

Tabla 1.

Frecuencia de utilización de recursos educativos (blogs, redes sociales, foros, etc.) por el alumnado a lo largo de su formación universitaria.

\begin{tabular}{lcc}
\hline & Frecuencia & Porcentaje \\
\hline Nunca & 13 & $\mathbf{3 8 , 2}$ \\
\hline Casi nunca & 8 & $\mathbf{2 3 , 5}$ \\
\hline Alguna vez & 8 & 23,5 \\
\hline Casi siempre & 2 & 5,9 \\
\hline Siempre & 3 & 8,9 \\
\hline Total & 34 & 100 \\
\hline
\end{tabular}

Fuente: Elaboración propia.

Igualmente, el desconocimiento por parte del alumnado de este tipo de recursos educativos quedó manifiesto, ya que, probablemente, por este motivo un alto porcentaje de alumnos consideró que para trabajar con el blog necesitaría formación previa. En cuanto al uso que los encuestados atribuían inicialmente a los blogs, éstos 
contemplaban que era, principalmente, educativo $(31,25 \%)$ y en menor medida, científico $(26,25 \%)$, escogiendo la mayoría de los alumnos respuestas múltiples entre las que también se incluían funciones sociales y personales (figura 1).

Llama la atención que, a pesar de que las generaciones conocidas como "nativos digitales" se caracterizan por su habilidad y aptitud para las nuevas tecnologías, se percibe que, al sacarles de su entorno digital habitual, normalmente relacionado con las redes sociales (Facebook, Instagram, Twitter, YouTube, etc.), manifiestan, en un primer momento, cierta inseguridad ante lo desconocido, lo cual provoca que se adopten actitudes reticentes ante lo que se les plantea, a pesar de presentar las destrezas y habilidades básicas necesarias.

\section{Finalidad del blog \%}

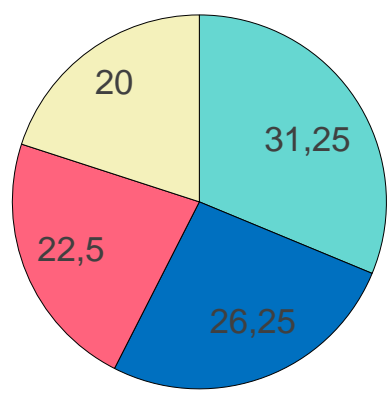

$\square$ Educativa $\square$ Científica $\square$ Social $\square$ Personal

Figura 1.

Visión previa del alumnado de la finalidad del blog como recurso.

Fuente: Elaboración propia.

Antes de utilizar el blog, en el cuestionario inicial, se les preguntó a los estudiantes acerca de las posibles ventajas que consideraban podría suponer el uso del mismo como recurso educativo, pudiendo encontrar respuestas, tales como: "es de fácil entrada, abarca diferentes campos dentro del mundo de la investigación y resulta altamente interesante" o por ejemplo: "Te obliga a trabajar sobre contenidos de la asignatura, te permite leer y aprender a través del trabajo de tus compañeros y es otra nueva forma de trabajar la cual ayuda a adquirir conocimientos con mayor facilidad", entre otras. En este sentido, tras la implementación del blog, los aspectos que los alumnos consideraron más útiles y/o positivos de la experiencia fueron: la reflexión y profundización de los temas tratados en la asignatura, así como el trabajo grupal y el debate final.

En contraposición, entre los inconvenientes destacados por los alumnos sobre la utilización del blog, además del ya citado desconocimiento a esta herramienta, fueron el miedo a las críticas entre compañeros, el esfuerzo extra dada la cantidad de prácticas y actividades de otras asignaturas y el tiempo y dedicación invertidos en su uso. Siendo interesante este último, ya que el $61,8 \%$ de los alumnos coincidieron en que la elaboración de las entradas les había requerido "bastante" dedicación como respuesta a su valoración personal acerca de la dedicación requerida para elaborar 
entradas y comentar la de los compañeros (tabla 2). Además de esto, es importante destacar que el $58,8 \%$ de los alumnos afirmó que el blog les había exigido mayor dedicación que otros métodos "tradicionales" de trabajo.

Tabla 2.

Valoración del alumnado de la dedicación requerida para elaborar la entrada del blog y comentar las de los compañeros y compañeras.

\begin{tabular}{lcc}
\hline & Frecuencia & Porcentaje \\
\hline Normal & 10 & 29,4 \\
\hline Bastante & 21 & $\mathbf{6 1 , 8}$ \\
\hline Excesiva & 3 & 8,8 \\
\hline Total & 34 & 100 \\
\hline
\end{tabular}

Fuente: Elaboración propia.

Respecto al aprendizaje de los contenidos de la asignatura, la respuesta por parte del alumnado al uso del blog fue muy positiva, ya que el $85,3 \%$ estaban "de acuerdo" o "totalmente de acuerdo" en que sería un recurso favorecedor previamente a su implementación. Esta opinión no solo se mantuvo, sino que mejoró significativamente al finalizar la actividad docente $(p=0,014)$, elevando el porcentaje hasta el $94,1 \%$ (tabla 3). Dicha mejora del aprendizaje se debió, entre otros motivos, al proceso de búsqueda y selección de información y posterior exposición de la misma, lo que les permitió adquirir y ampliar sus conocimientos, no solo con la publicación de sus entradas, sino con la interacción con el resto de los compañeros y compañeras.

En lo referente a la metodología utilizada, los resultados son similares a lo observado en el aprendizaje, ya que, nuevamente, un porcentaje muy elevado $(73,5 \%)$ estaba "de acuerdo" o "totalmente de acuerdo" en que el uso del blog grupal mejoraría la metodología de la asignatura, incrementándose significativamente este porcentaje tras la implementación del mismo $(p=0,033)$, hasta el $94,1 \%$ (tabla 3$)$. Dichos resultados se fundamentaron en la amplitud y variedad de nuevos recursos ofrecidos por el blog (otros blogs de divulgación científica, páginas de interés, artículos científicos, etc.), lo cual les permitió aclarar y ampliar los contenidos trabajados en la asignatura.

Tabla 3.

Comparación de las opiniones del alumnado entre la encuesta inicial y final acerca del uso del blog sobre el aprendizaje y la metodología. Prueba de rangos con signo Wilcoxon $(p \leq 0,05)$.

\begin{tabular}{|c|c|c|c|c|}
\hline & Aprendizaje $0^{a}$ & Aprendizaje $1^{b}$ & Metodología $0^{c}$ & Metodología $1^{d}$ \\
\hline & \multicolumn{2}{|c|}{ Porcentaje } & \multicolumn{2}{|c|}{ Porcentaje } \\
\hline En desacuerdo & 2,9 & 2,9 & 0 & 0 \\
\hline Neutral & 11,8 & 2,9 & 26,5 & 5,9 \\
\hline De acuerdo & 70,6 & 44,1 & 52,9 & 58,8 \\
\hline $\begin{array}{l}\text { Totalmente de } \\
\text { acuerdo }\end{array}$ & 14,7 & 50,1 & 20,6 & 35,3 \\
\hline Total & 100 & 100 & 100 & 100 \\
\hline Valor $p \leq 0,05$ & \multicolumn{2}{|c|}{0,014} & \multicolumn{2}{|c|}{0,033} \\
\hline
\end{tabular}

a. ¿Crees que el uso del Blog favorecerá el aprendizaje?

b. ¿Crees que el Blog ha favorecido tu aprendizaje de los contenidos de la asignatura?

c. ¿Crees que el uso del Blog favorecerá la metodología?

d. ¿Crees que el Blog ha mejorado la metodología empleada en la asignatura?

Fuente: Elaboración propia.

Los resultados obtenidos refuerzan la importancia de los blogs colaborativos en el ámbito educativo, ya que ponen de manifiesto que se trata de una práctica adecuada y útil que favorece un papel activo del estudiante, al fomentar habilidades y competencias de orden superior, así como el desarrollo de metodologías de 
aprendizaje cooperativo, de acuerdo con lo descrito por O'Donnell (2006). Por otro lado, los resultados evidencian la utilidad de desarrollar competencias de autoevaluación, coincidiendo con lo sugerido por la investigación educativa desarrollada por Carneiro y cols. (2011) en relación con la clara mejora de los resultados, favorecida por una participación activa en el seguimiento de los procesos de aprendizaje

Por otro lado, se observó que a gran parte de los encuestados el trabajo con el blog le había sido útil a la hora de asimilar los contenidos de la asignatura. En este sentido, el 82,4\% de los estudiantes estaba "de acuerdo" o "totalmente de acuerdo" en que el blog les había ayudado a asimilar los contenidos de la asignatura mejor que otras metodologías "tradicionales" (monografías, seminarios, presentaciones, etc.) (tabla 4). Algunos de los motivos dados por los encuestados para esta cuestión fueron: "podemos aclarar y ampliar el conocimiento de la materia impartida en clase"; "porque los profesores pueden hacerse una idea de nuestro conocimiento e interés" o "porque las TIC están a la orden del día y nos ayudan", entre otros.

Tabla 4.

Valoración del alumnado sobre la asimilación los contenidos de la asignatura con el blog en comparación con los métodos tradicionales.

\begin{tabular}{lcc}
\hline & Frecuencia & Porcentaje \\
\hline Neutral & 6 & 17,6 \\
\hline De acuerdo & 12 & $\mathbf{3 5 , 3}$ \\
\hline Totalmente de acuerdo & 16 & $\mathbf{4 7 , 1}$ \\
\hline Total & 34 & 100 \\
\hline
\end{tabular}

Fuente: Elaboración propia.

Por último, los resultados obtenidos muestran que el uso del blog grupal aumentó la motivación hacia los contenidos de la asignatura, incluso más que otros métodos "tradicionales" de trabajo. De acuerdo con las respuestas del alumnado, el blog les pareció una metodología novedosa y atractiva que rompía con la monotonía, la cual puede desembocar en una pérdida de motivación. Al mismo tiempo, queda patente que esta experiencia hizo más interesante y útil el contenido de la asignatura, ya que la exposición pública de conocimientos y opiniones, así como el debate posterior con el resto de los compañeros y compañeras, fomentó de forma exponencial el interés por parte del alumnado y el aprendizaje de los diferentes temas tratados.

\section{CONCLUSIONES}

El presente estudio evidencia que el uso de los blogs colaborativos como instrumento educativo, promueve la autorregulación por medio de actividades de coevaluación y/o autoevaluación, a través de las cuales el alumnado construye su propio proceso de aprendizaje. Además, han demostrado ser un excelente medio para el desarrollo de la competencia digital. En este sentido, los alumnos aprenden a buscar, obtener, filtrar, procesar y comunicar información para transformarla en conocimiento.

Por otro lado, y a pesar del rechazo inicial a que el trabajo fuera evaluado por los compañeros, el uso del blog grupal como herramienta de aprendizaje ha permitido estimular y fomentar las habilidades comunicativas del alumnado. No obstante, como posible limitación, cabría destacar que los alumnos suelen seguir únicamente las 
indicaciones del profesor, excluyendo en muchos casos la espontaneidad tanto en la participación, como en su expresión. Por ello, las líneas de mejora se deben centran en incrementar la participación y motivación del alumnado probando diferentes estrategias (entradas cortas, unidades de contenido, etc.) para mejorar la implementación del blog como recurso educativo.

Todo ello, pone de manifiesto las múltiples posibilidades educativas que ofrecen los blogs colaborativos en el ámbito didáctico, mostrándose como una de las herramientas que mejor se adecúan a la implementación y desarrollo de estrategias e instrumentos de autorregulación.

\section{REFERENCIAS}

Brown, S., \& Glasner, A. (2003). Evaluar en la universidad. Problemas y nuevos enfoques. Madrid: Narcea.

Carneiro, R., Lefrere, P., Steffens, K., \& Underwood, J. (2011) Self-regulated Learning in Technology Enhanced Learning Environments: A European Perspective. The Nether lands: Sense Publishers.

Conde-Caballero, D., Castillo, C. A., Ballesteros-Yáñez, I., \& Mariano-Juárez, L. (2019). Blogging as a tool for the acquisition and dissemination of knowledge in health sciences: a preliminary evaluation. International Journal of Educational Technology in Higher Education, 16(1), 1-15.

Darby, J. (2007). Evaluating course evaluations: the need to establish what is being measured. Assessment y Evaluation in Higher Education, 32(4), 441-455.

Delgado, V., Hortigüela, D., Ausín, V., \& Abella, V. (2018). El Blog como Instrumento de Mejora para la Autorregulación del Aprendizaje del Estudiante Universitario. Estudios pedagógicos (Valdivia), 44(2), 171-184.

Gamliel, E., \& Davidovitz, L. (2005). Online versus traditional teaching evaluation: mode can matter. Assessment y Evaluation in Higher Education, 30(6), 581592.

González, I. (2006). Dimensiones de la evaluación en el Espacio Europeo de Educación Superior. Electronic Journal of Research in Educational Psychology, 4(3), 445-468.

González, L. (2003). Aproximación a una formación académica de calidad: el punto de vista de los estudiantes universitarios. Electronic Journal of Research in Educational Psychology, 1(1), 20-42.

Huffaker, D. (2005). The educated blogger: Using weblogs to promote literacy in the classroom. AACE Journal, 13(2), 91-98.

Jackson, N., \& Ward, R. (2004). A fresh perspective on progress files - a way of representing complex learning and achievement in higher education. Assessment y Evaluation in Higher Education, 29(4), 423-449.

O'Donnell, M. (2006). Blogging as pedagogic practice: artefact and ecology. Asia Pacific Media Educator, 17, 5-19.

Stowell, M. (2004). Equity, justice and standards: assessment decision making in higher education. Assessment y Evaluation in Higher Education, 29(4), 495510. 